\title{
Effect of pressurizing speed on filling behavior of gradual expansion structure in low pressure casting of ZL 205A alloy
}

\author{
*Shan-guang Liu, Chuan-biao Luo, Guo-ai Li, Wen-lin Gao, Zheng Lu, Sheng-long Dai \\ Beijing Institute of Aeronautical Materials, Beijing 100095, China
}

\begin{abstract}
The mold filling behavior of gradual expansion structure in low pressure casting was studied by two phase flow model using the Volume of Fluid method, and was verified by water simulation with a Plexiglas mold. To get smooth mold filling process and provide a guide for the pressurizing speed design in the producing practice, the mathematical model with the pressurizing speed, expansion angle and height of the gradual expansion structure was established. For validation experiments, ZL205A alloy castings were produced under two different pressurizing speeds. Weibull probability plots were used to assess the fracture mechanisms under different pressurizing speeds. Mechanical properties of ZL205A alloy were applied to assess the entrainment of oxide film. The results show that the filling process of a gradual expansion structure in a low pressure casting can be divided into the spreading stage and filling stage by gate velocity. The gate velocity continues to increase in the gradual expansion structure, and increases with the increase of pressurizing speed or expansion angle. Under the effect of the falling fluid raised by the jet flow along the sidewall, the fluid velocity decreases in the jet zone from ingate to free surface. As such, oxide film entrainment does not occur when the gate velocity is greater than the critical velocity, andthe gate velocity no longer reflects the real state of the free surface. The scatter of the mechanical properties is strongly affected by the entrainment of oxide films.
\end{abstract}

Key words: low pressure casting; two phase flow; water simulation; oxide film; ZL205A alloy

CLC numbers: TG146.21 Document code: A Article ID: 1672-6421(2018)04-276-07

$\mathrm{T}$ he liquid aluminum alloy surface is always covered with a layer of oxide film. If the surface oxide film is broken, a new thin oxide film will form immediately, then it will become thicker. However, the broken oxide film can be entrained in the bulk liquid by the surface turbulence, and some large thick oxide films are easily detected by non-destructive testing and microscopy in the finished castings ${ }^{[1]}$. The surface oxide film can be folded to double layers in the entrainment process, and the bonding between the opposed interfaces is negligible, causing the defect to then resemble and act as a $\operatorname{crack}^{[2]}$. Recent studies announced that the double oxide film defects, entrained during the filling process when the velocity of liquid aluminum is greater than a critical gating velocity, are the main reason for the failure of aluminum castings ${ }^{[3-5]}$. Runyoro ${ }^{[6]}$ and Bahreinian ${ }^{[7]}$ indicated the critical velocity is $0.5 \mathrm{~m} \cdot \mathrm{s}^{-1}$ in the bottom gating system.

\section{*Shan-guang Liu}

Male, born in 1983, senior engineer, Ph.D. Research interests: development and casting technology of aluminum alloy.

E-mail: liusg621@126.com

Received: 2018-03-27; Accepted: 2018-04-12
Low pressure casting (LPC) is regarded as an effective solution to avoid the entrainment of oxide film ${ }^{[8]}$. However, Liu et al. ${ }^{[9]}$ demonstrated that the oxide film could be entrained during the filling process of a sudden expansion structure at an unreasonable pressurizing speed in LPC process, which is also the case of sudden contraction structure in $\mathrm{LPC}^{[10]}$. It is obvious that the filling behavior and inclusions in LPC are influenced not only by pressurizing speed but also by the variation of cross-section of cavity.

In the present work, the filling behavior of a plateshaped casting with a gradual expansion structure in LPC was studied by the experiment and simulation analysis. The relationship among the pressurizing speed, expansion angle and height of the gradual expansion structure was established, and the mechanical properties of ZL205A alloy was applied to assess the entrainment of oxide film.

\section{Experimental procedures}

\subsection{Computational modeling}

A computational fluid dynamics (CFD) code, FLUENT, was employed in this work and the filling process was 
simulated by the VOF (Volume of Fluid) algorithm of two-phase flow model. To visually verify the numerical simulation results, water was used as the filling medium. The physical data of water used in numerical simulation are listed in Table 1.

Table 1: Physical data of water used for numerical simulation

\begin{tabular}{cccc}
$\begin{array}{c}\text { Density } \\
\left(\mathrm{kg}^{-3}\right)\end{array}$ & $\begin{array}{c}\text { Contact angle } \\
\left({ }^{\circ}\right)\end{array}$ & $\begin{array}{c}\text { Surface tension } \\
\text { coefficient } \\
\left(\mathbf{N} \cdot \mathbf{m}^{-1}\right)\end{array}$ & $\begin{array}{c}\text { Dynamic } \\
\text { viscosity } \\
(\mathbf{P a} \mathbf{s})\end{array}$ \\
\hline 998 & 160 & 0.072 & 0.000839 \\
\hline
\end{tabular}

\subsection{Water simulation test}

According to the principle of similarity theory, dynamic similarity is the primary factor affecting the kinematic similarity. In this work, the principal similarity criterion is Reynolds:

$$
R e_{\mathrm{a}}=R e_{\mathrm{w}}
$$

where the $R e$ is a dimensionless number, called Renault number and the suffix "a" and "w" stand for Al alloy and water model, respectively.

Water simulation was observed and the filling behavior was recorded by particle image velocimetry (PIV). The PIV, as a transient flow field measurement technique, has recently been used in a wide variety of fields, the measurement system of which consists of a double pulse laser, charge coupled device (CCD camera), personal computer (PC) and software. The double pulse laser is a combination of a pair of Neodymiumdoped yttrium Aluminum garnet (Nd-YAG) lasers. The details of the arrangement of the PIV system and the measurement procedure were described by Kawaguchi ${ }^{[1]}$.

Low pressure casting machine usually includes bottom vessel and upper and lower computer, in which the computer is used to control the pressure in the bottom vessel. The filling medium was housed inside the bottom vessel, and the plexiglass mold was placed on the bottom vessel with a riser tube. The surface of the filling medium in the bottom vessel is pressed by gas, then the filling medium was forced to rise through the riser tube and consequently fed the plexiglass mold. The filling process was recorded by the PIV system. The arrangement of the low pressure casting equipment and PIV system is shown in Fig. 1.

\subsection{Casting experiment}

A commercial aluminum alloy ZL205A (the chemical

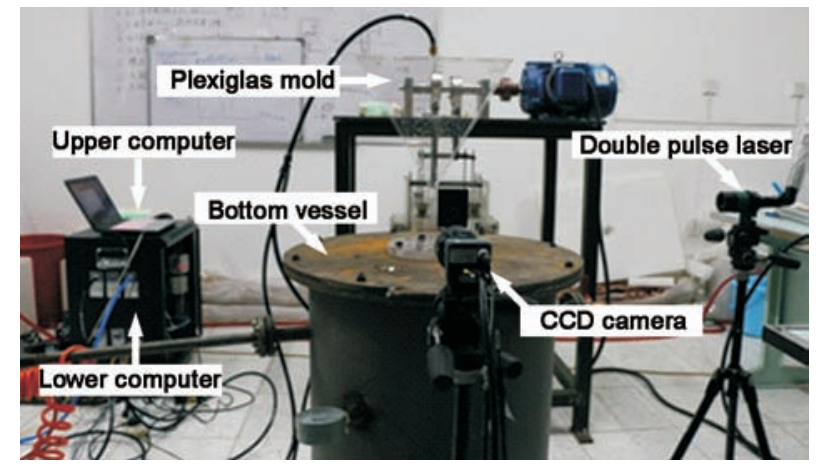

Fig. 1: Equipment diagram of water simulation test
Table 2: Chemical composition of ZL205A alloy (wt.\%)

$\begin{array}{cccccccc}\mathrm{Cu} & \mathrm{Mn} & \mathrm{Ti} & \mathbf{V} & \mathrm{Zr} & \mathrm{Cd} & \mathrm{B} & \mathrm{Al} \\ 5.05 & 0.40 & 0.23 & 0.20 & 0.10 & 0.18 & 0.010 & \text { Bal. }\end{array}$

composition is shown in Table 2) was melted in an electrical furnace and the melting temperature was kept at $(750 \pm 10){ }^{\circ} \mathrm{C}$ for $30 \mathrm{~min}$. The melted liquid was degassed by bubbling pure argon $(99.99 \%)$ into the melt for 20 min using rotary degassing at $(720 \pm 5)^{\circ} \mathrm{C}$, then poured into a resin-bonded sand mold with an expansion angle of $30^{\circ}$ at $(710 \pm 5)^{\circ} \mathrm{C}$.

\subsection{Tensile property test}

Tensile samples with a diameter of $10 \mathrm{~mm}$ were machined from the casting. The dimensions of the tensile samples are shown in Fig. 2. Tensile tests were performed using a INSTRON5569 material test machine at room temperature and a constant drawing speed of $1 \mathrm{~mm} \cdot \mathrm{min}^{-1}$.

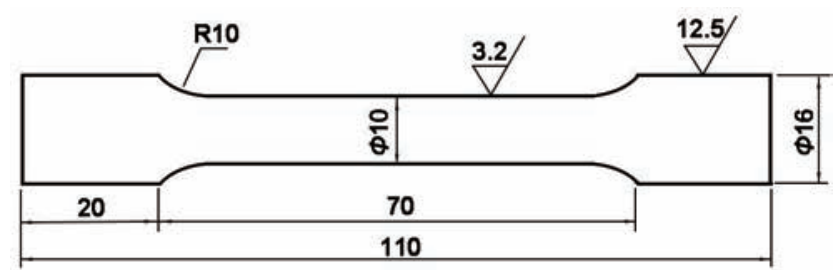

Fig. 2: Schematic diagram of room temperature tensile sample (all dimensions in $\mathrm{mm}$ )

The purpose of this work is to evaluate the effect of surface turbulence on the reliability of castings, therefore, all the samples were tested without heat treatment.

\subsection{Weibull statistical analysis}

Campbell et al. ${ }^{[12]}$ proved that the Weibull distribution can be used to predict the probability of fracture. The cumulative probability function of the Weibull distribution $(P i)$ is determined by the following equation:

$$
P i=1-\exp \left[-\left(\frac{\sigma-\sigma_{\mathrm{T}}}{\sigma_{0}}\right)^{m}\right]
$$

where $\sigma$ is the given stress; $\sigma_{\mathrm{T}}$ is the threshold value below which no specimen is expected to fail; $\sigma_{0}$ is the scale parameter; and $m$ is the shape parameter often referred to as Weibull modulus.

It can be plotted as a straight line by re-arranging Eq. (2), as follows:

$$
\ln [-\ln (1-P i)]=m \ln \left(\sigma-\sigma_{\mathrm{T}}\right)-m \ln \left(\sigma_{0}\right)
$$

When the left-hand side of the equation is plotted as a function of $\ln \left(\sigma-\sigma_{\mathrm{T}}\right)$, a linear form of three-parameter Weibull plot with the slope of $m$ and an intercept of $m \ln \left(\sigma_{0}\right)$ could be derived. If the threshold value $\sigma_{\mathrm{T}}$ is zero, a two-parameter plot then is obtained. 


\section{Results and discussion}

\subsection{VOF simulation results}

Three physical models of gradual expansion structure with different expansion angles $\left(30^{\circ}, 45^{\circ}\right.$ and $\left.60^{\circ}\right)$ were designed to study the effect of expansion angle on the filling process in LPC, as shown in Fig. 3.
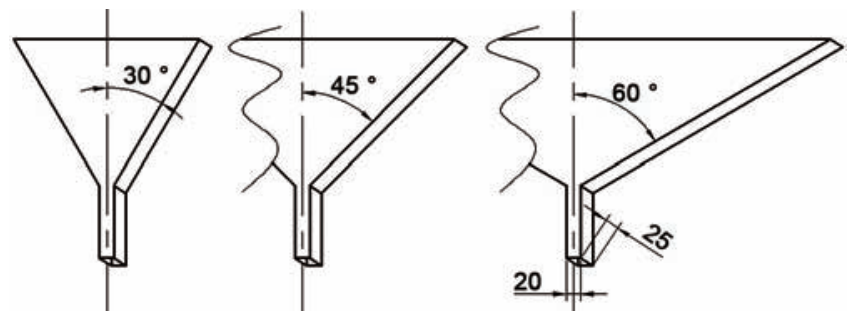

Fig. 3: Schematic diagram of gradual expansion structure with different expansion angles (unit: $\mathrm{mm}$ )

The average velocity of a cross section of riser tube (called "gate velocity") during the mold filling process was recorded in FLUENT software. Figure 4 shows the gate velocity of the gradual expansion structure with different expansion angles under a pressurizing speed of $1,000 \mathrm{~Pa} \cdot \mathrm{s}^{-1}$. The mold filling process of the gradual expansion structure with different expansion angles under a certain pressurizing speed can be divided into raising stage (characterized by the damping oscillations), spreading stage (characterized by the dramatic increase of velocity) and filling stage (characterized by the increase of velocity with relative lower accelerated speed) according to the change rules of the gate velocity, this phenomenon was also observed in Liu's work ${ }^{[9]}$. The gate velocity increases with the expansion angle under a constant pressurizing speed and has the same tendency during the filling process of the gradual expansion structure with different expansion angles.

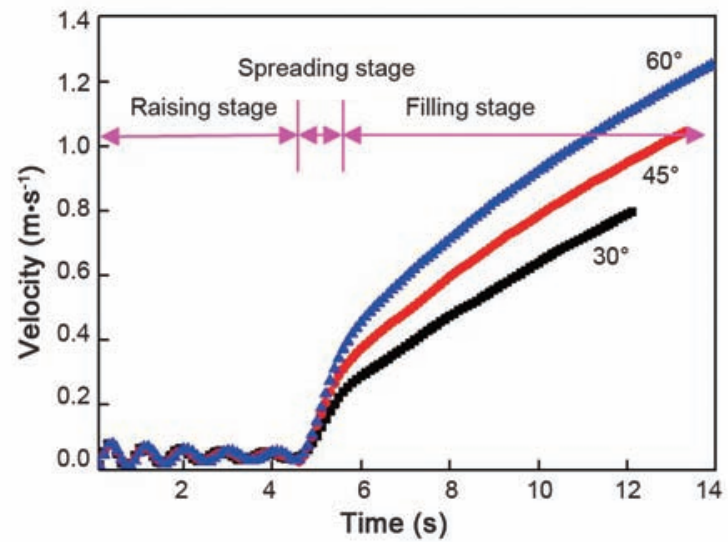

Fig. 4: Gate velocity of gradual expansion structure with different expansion angles under $1,000 \mathrm{~Pa} \cdot \mathrm{s}^{-1}$

The gradual expansion structure with the expansion angle of $30^{\circ}$ was adopted to illustrate the effect of pressurizing speed on gate velocity. Figure 5 shows that the gate velocity of the gradual expansion structure with a constant expansion angle of $30^{\circ}$ under different pressurizing speeds. The gate velocity increased during the filling process with the increase of pressurizing speeds, and also had the same tendency under different pressurizing speeds.

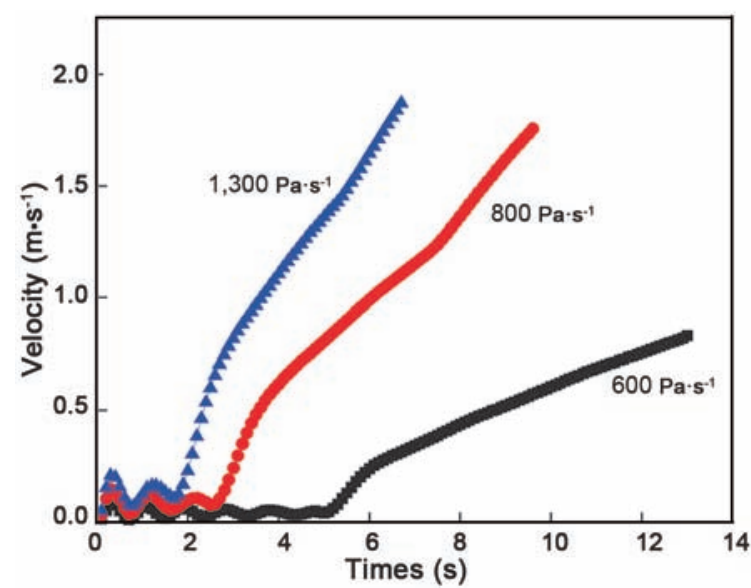

Fig. 5: Gate velocity of gradual expansion structure with expansion angle of $30^{\circ}$ under different pressurizing speeds

The filling behavior and velocity field under different pressurizing speeds in the gradual expansion structure with the expansion angle of $30^{\circ}$ are shown in Fig. 6. The velocity vector diagram shows that there is a jet zone above the ingate during the filling process under different pressurizing speeds, as shown in Fig. 6(a2). At the beginning of the filling stage, the free surface remained smooth under different pressurizing speeds [Fig. 6(a1) at $7.4 \mathrm{~s},(\mathrm{~b} 1)$ at $3.4 \mathrm{~s}$ and (c1) at $1.4 \mathrm{~s}$ ] due to relatively low gate velocity and inability of oxide film entrainment.

With the increase of the pressurizing speed, the free surface becomes less smooth. The free surface of the liquid exhibits convex curvature under the effect of the jet and the raising liquid falls back due to the gravity resulting into falling velocity, as shown in Fig. 6(b2). The falling velocity is the main reason for entrainment of air or oxide film.

The gate velocity kept increasing during the filling process in the gradual expansion structure, which caused the increase of the jet intensity. When the pressurizing speed was 600 $\mathrm{Pa} \cdot \mathrm{s}^{-1}$, the falling velocity of the liquid near the free surface was low (lower than $0.1 \mathrm{~m} \cdot \mathrm{s}^{-1}$ ) and the free surface was almost smooth, as shown in Fig. 6(a2) and (a3). Nevertheless, in the case when the pressurizing speed rose to $800 \mathrm{~Pa} \cdot \mathrm{s}^{-1}$, the falling velocity were greater than $0.2 \mathrm{~m} \cdot \mathrm{s}^{-1}$ in Fig. 6(b3). The radius of curvature of the free surface above the jet zone decreased and the free surface was no longer smooth. In other words, the surface turbulence took place. This means that the filling process is not steady and the entrainment of surface oxide film was occurred. For the pressurizing speed of $1,300 \mathrm{~Pa} \cdot \mathrm{s}^{-1}$, similar results were obtained, as shown in Fig. 6(c2) and (c3).

According to the balance of the inertia force and the surface tension, Campbell ${ }^{[2]}$ suggested that the critical velocity of water was $0.33 \mathrm{~m} \cdot \mathrm{s}^{-1}$ in the bottom gating system of gravity 


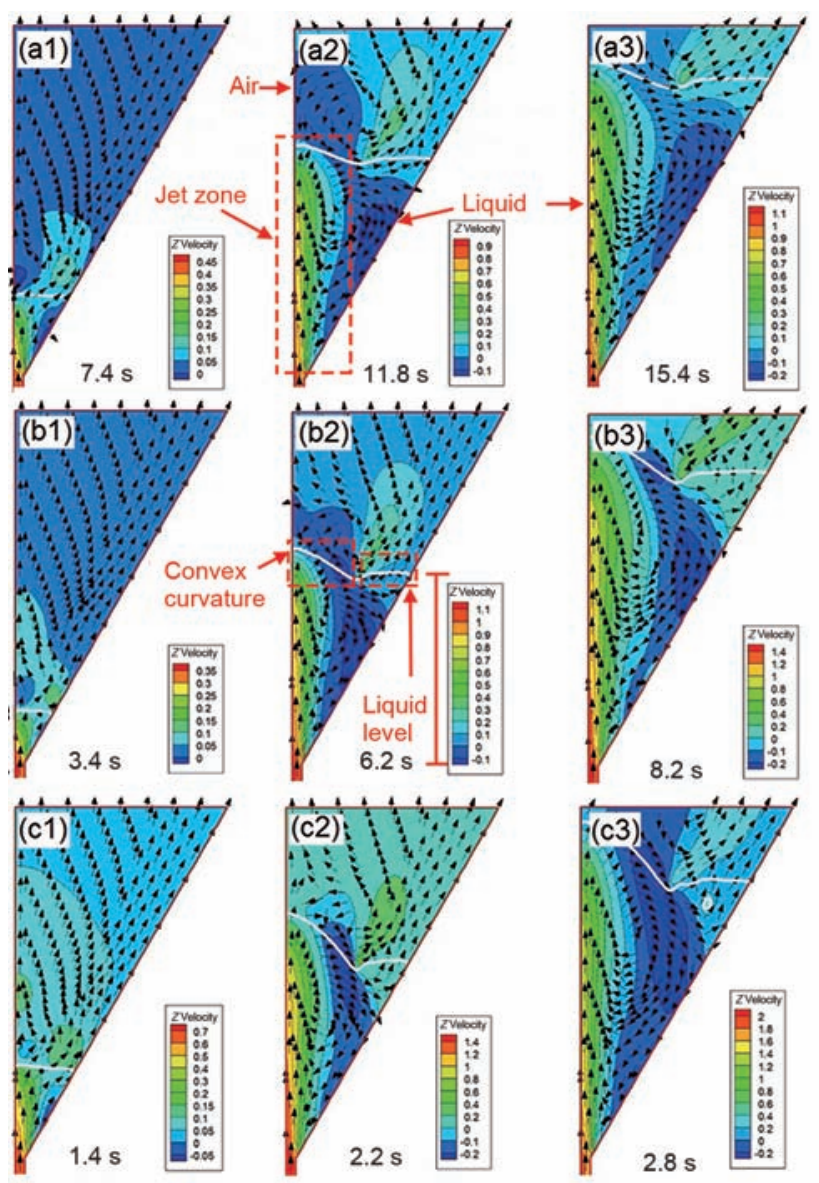

Fig. 6: Velocity field of mold filling under different pressuring speeds in gradual expansion structure: (a) $600 \mathrm{~Pa} \cdot \mathrm{s}^{-1}$, (b) $800 \mathrm{~Pa} \cdot \mathrm{s}^{-1}$, (c) 1,300 Pa $\cdot \mathrm{s}^{-1}$ casting. Liu et al. ${ }^{[13]}$ pointed out that the critical velocity $\left(0.33 \mathrm{~m} \cdot \mathrm{s}^{-1}\right)$ is also applicable to vacuum suction casting in the filling process of the sudden expansion structure. The main reason is that the velocity of the fluid, in the jet zone from ingate to free surface, remains almost unchanged in the filling process of the sudden expansion structure. It can be considered that the inertia force of the fluid in the ingate acts on the liquid free surface directly. Therefore, the oxide film entrainment could not occur when the gate velocity is lower than the critical velocity.

However, during the filling process of the gradual expansion structure in the low-pressure casting, the velocity of the fluid kept decreasing in the jet zone from ingate to the free surface. The reason is that the falling fluid is raised by the jet flow along the sidewall of the gradual expansion structure, resulting in a downward velocity component which acts on the jet zone and lowers the velocity. Therefore, the velocity of the fluid reaching the height of the liquid level near the free surface was less than the critical velocity, although the gate velocity was greater than that. Obviously, in this case, the gate velocity itself no longer determines whether the entrainment of oxide film occurs. The entrainment is affected by the action of gate velocity, expansion angle and the height of liquid level.

\subsection{Water simulation test}

In order to validate the VOF simulation results, a PIV system was employed to observe the filling behavior in LPC. Figure 7 shows the filling process of water simulation under different pressurizing speeds in a gradual expansion structure. When
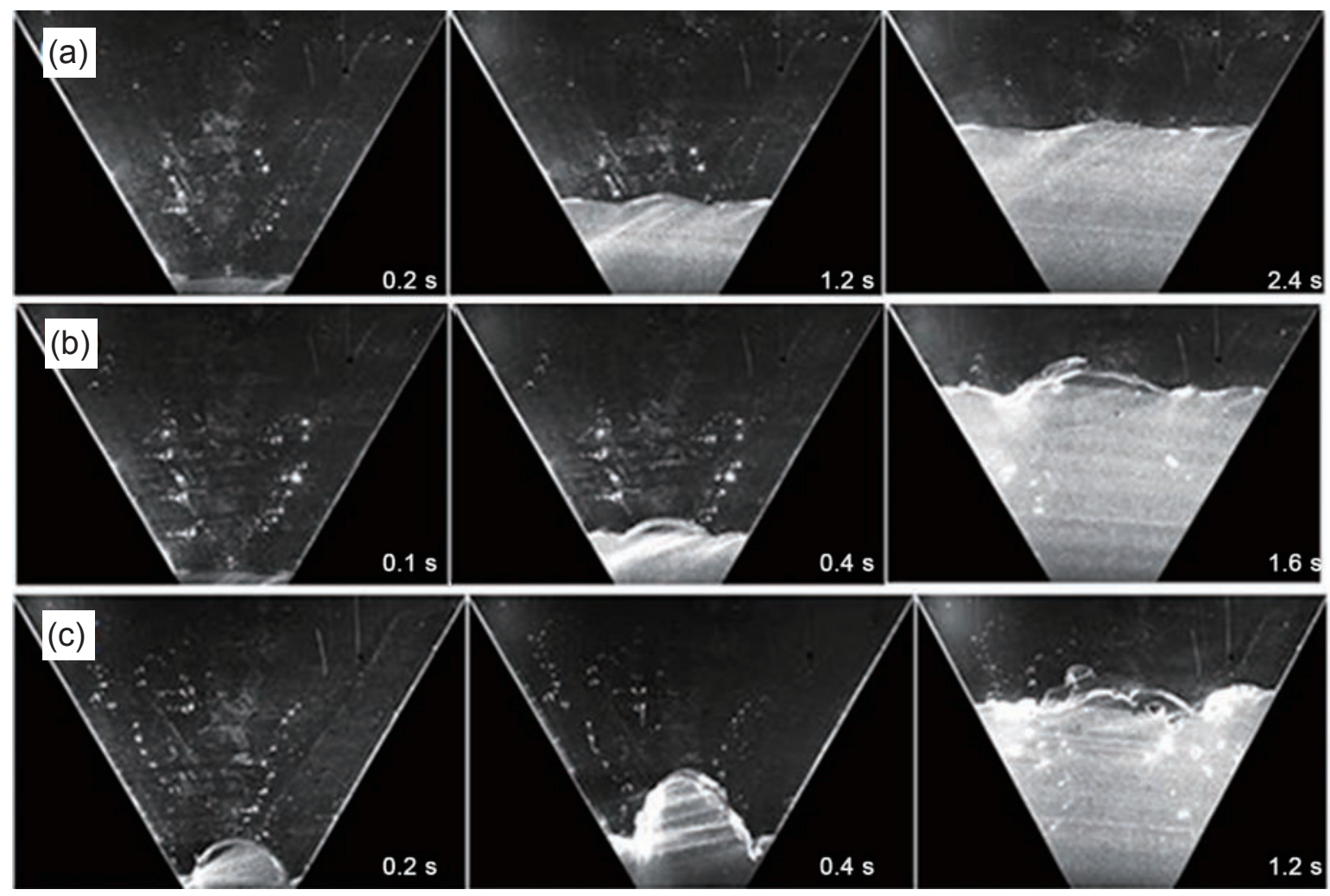

Fig. 7: Water simulation results of entrainment behaviors in gradual expansion structure under different pressurizing speeds: (a) $633 \mathrm{~Pa} \cdot \mathrm{s}^{-1}$, (b) $794 \mathrm{~Pa} \cdot \mathrm{s}^{-1}$, (c) 1,270 $\mathrm{Pa} \cdot \mathrm{s}^{-1}$ 
the pressurizing speed was $633 \mathrm{~Pa} \cdot \mathrm{s}^{-1}$, the filling process was steady and smooth, as shown in Fig. 7(a). While the pressurizing speed was $794 \mathrm{~Pa} \cdot \mathrm{s}^{-1}$, the free surface remained smooth in the initial stage of the filling process (almost the filling stage). During the subsequent filling process [at $0.4 \mathrm{~s}$ in Fig. 7(b)], the free surface of the liquid exhibited a convex curvature, and the air entrainment was not observed. However, bubbles were observed at $1.6 \mathrm{~s}$, which means the air entrainment occurred (Actually, air entrainment in water corresponds to oxide film entrainment in Al alloys in this work). Similar results were obtained when the pressurizing speed was $1,270 \mathrm{~Pa} \cdot \mathrm{s}^{-1}$, as shown in Fig. 7(c). The intensity of surface turbulence increasing with the filling time is attributed to the increasing gate velocity during the filling process of the gradual expansion structure in LPC.

\subsection{Pressurizing speed design}

When the expansion angle of a gradual expansion structure reached $90^{\circ}$, the gradual expansion structure transformed to the sudden expansion structure. Furthermore, the filling behavior of the gradual expansion structure was close to that of the sudden expansion structure when the expansion angle was near $90^{\circ}$ due to the reducing influence of the sidewalls on the falling fluid. Therefore, it is reasonable to take the sudden expansion structure as a special form of gradual expansion structure. The method for determining the reasonable pressurizing speed in a sudden expansion structure is ${ }^{[9]}$ :

$$
\frac{\mathrm{d} P(t)}{\mathrm{d} t}=\rho g\left(1+\frac{A_{\mathrm{c}}}{A_{\mathrm{F}}}\right) \frac{A_{\mathrm{f}}}{A_{\mathrm{c}}} v_{\mathrm{g}}
$$

where $A_{\mathrm{F}}$ is the cross-section area of crucible $\left(\mathrm{m}^{2}\right) ; A_{\mathrm{f}}$ is the cross-section area of raiser tube $\left(\mathrm{m}^{2}\right) ; A_{\mathrm{c}}$ is the crosssection area of mold cavity $\left(\mathrm{m}^{2}\right) ; g$ is the gravity acceleration $\left(\mathrm{m} \cdot \mathrm{s}^{-2}\right) ; \rho$ is the density of liquid $\left(\mathrm{Kg} \cdot \mathrm{m}^{-3}\right) ; v_{\mathrm{g}}$ is the critical velocity $\left(\mathrm{m} \cdot \mathrm{s}^{-2}\right) ; P(t)$ is the pressure function of time $(\mathrm{Pa}) ; t$ is time (s). The units of measurement used in this work are international standard measurement units.

Due to the gate velocity continuing to increase during the filling process of the gradual expansion structure, the gate velocity will reach to the maximum value when the liquid level advances to the maximum cross-section area of the gradual expansion structure. Just to ensure the oxide film entrainment does not occur at the maximum cross-section area of the gradual expansion structure, the oxide film entrainment will be avoided during the whole filling process. In the case of the gradual expansion structure, the $H_{\mathrm{h}}(\mathrm{m})$ is the height of the gradual expansion structure, and also represents the height of the liquid level where the oxide film entrainment occurrs, $A_{\mathrm{d}}$ is the cross-section area $\left(\mathrm{m}^{2}\right)$ and $B_{\mathrm{b}}$ is the thickness of the gradual expansion structure $(\mathrm{m}), \theta$ is the expansion angle $\left(^{\circ}\right)$. The cross-section area at the top of gradual expansion structure can be expressed as:

$$
A_{\mathrm{d}}=A_{\mathrm{f}}+H_{\mathrm{h}} \cdot B_{\mathrm{b}} \cdot \tan \theta
$$

Replacing the $A_{\mathrm{c}}$ in Eq. (4) with $A_{\mathrm{d}}$ in Eq. (5), the pressurizing speed in gradual expansion structure is expressed as follows:

$$
\frac{\mathrm{d} P(t)}{\mathrm{d} t}=\rho g\left(\frac{A_{\mathrm{f}}}{A_{\mathrm{f}}+H_{\mathrm{h}} \cdot B_{\mathrm{b}} \cdot \tan \theta}+\frac{A_{\mathrm{c}}}{A_{\mathrm{F}}}\right) v_{\mathrm{g}}
$$

From the Eq. (6), the pressurizing speed is influenced by the expansion angle and the height of the gradual expansion structure besides the cross-section areas of furnace and riser tube. In practice, the pressurizing speed is sensitive to the expansion angle and the height of the gradual expansion structure, as the cross-section areas of furnace and riser tube are normally constants for the LPC equipment.

In order to prove the accuracy of Eq. (6), the height of air entrainment was measured under different pressurizing speeds in the gradual expansion structure with an expansion angle of $30^{\circ}$ by water simulation test, and the comparison result is shown in Fig. 8. The results of the calculation and the experiment suggested that the height of oxide film entrainment occurrence increased with the decrease of the pressurizing speed. Although the error of the results between the experiment and calculation was $50 \mathrm{~mm}$ when the pressurizing speed was $600 \mathrm{~Pa} \cdot \mathrm{s}^{-1}$, this value decreased to $2 \mathrm{~mm}$ when the pressurizing speed was 2,000 $\mathrm{Pa} \cdot \mathrm{s}^{-1}$. The computational results of our model were in satisfactory agreement with the experimental results in high pressurizing speed, which showed the validity of the models in the work.

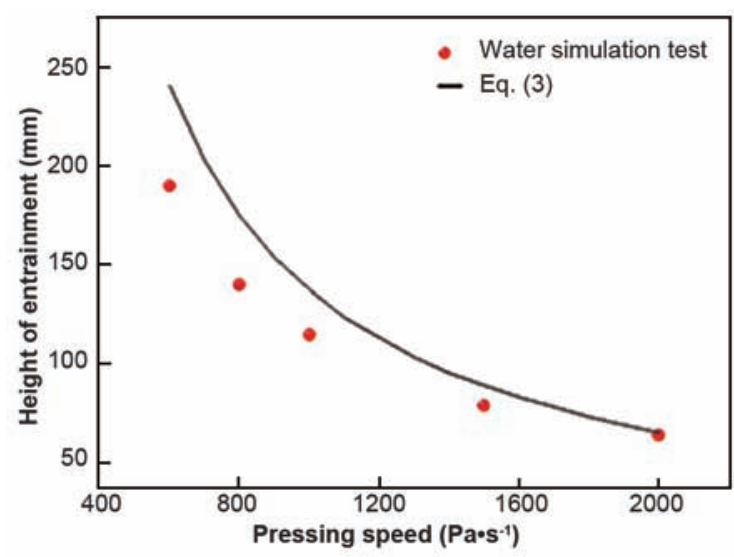

Fig. 8: Results comparison between water simulation test and Eq. (3) during filling process of gradual expansion structure with expansion angle of $30^{\circ}$

According to Eq. (6), the relationship of pressurizing speed, expansion angle and the height of oxide film entrainment showed in Fig. 9. The 3D surface denoted the critical state, above which the oxide film would be entrained. In practice, the dimensions of a casting are well known in that the height and expansion angle are known, consequently, the pressurizing speed should be chosen below the 3D surface to avoid the entrainment of oxide film. The calculation results suggested that the height of oxide film entrainment occurrence increased with the decreasing of the pressurizing speed and expansion angle. For the gradual expansion structure, lower pressurizing speed and expansion angle reduce the gate velocity during the filling process, as shown in Fig. 5 and Fig. 4, respectively. The 
velocity near liquid level, decreasing with the gate velocity, lower than critical velocity, may ensure a smooth filling process and avoid oxide film entrainment.

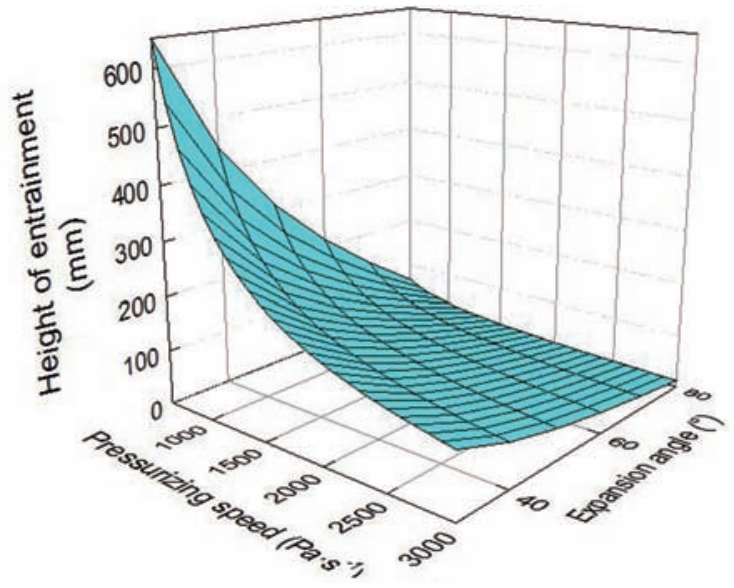

Fig. 9: Relationship of pressurizing speed, expansion angle and height of oxide film entrainment

\subsection{Casting experiment results}

Equation (5) has been proved by water test and numerical simulation results. In order to further confirm the validity of this mode, two ZL205A alloy castings of gradual expansion structure also with the expansion angle of $30^{\circ}$ were produced, and the pressurizing speed was designed by Eq. (5). According to Eq. (5), the critical pressurizing speed is $1,800 \mathrm{~Pa} \cdot \mathrm{s}^{-1}$ when the sand mold height is $200 \mathrm{~mm}$ and the expansion angle is $30^{\circ}$. Therefore, two pressurizing speeds $\left(1,500 \mathrm{~Pa} \cdot \mathrm{s}^{-1}\right.$ and 2,000

$\left.\mathrm{Pa} \cdot \mathrm{s}^{-1}\right)$ were applied to the casting experiment.

According to the ultimate tensile strengths three-parameter Weibull distributions plot was constructed and is shown in Fig. 10. $\mathrm{R}^{2}$ is the determination coefficient, which usually is used to evaluate the fitting results. The closer the value of $\mathrm{R}^{2}$ is to 1 , the higher the fitting degree. Based on the $\mathrm{R}^{2}$ values of curve fitting, three-parameter plots were both suitable for the two pressurizing speeds. The threshold values of the castings under 1,500 $\mathrm{Pa} \cdot \mathrm{s}^{-1}$ and 2,000 Pa.s ${ }^{-1}$ were 189.3 $\mathrm{MPa}$ and 165.6 MPa, respectively.

The typical fracture morphologies with different pressurizing speeds were observed by SEM to explore the fracture mechanism, as shown in Fig. 11. Shrinkage cavity was

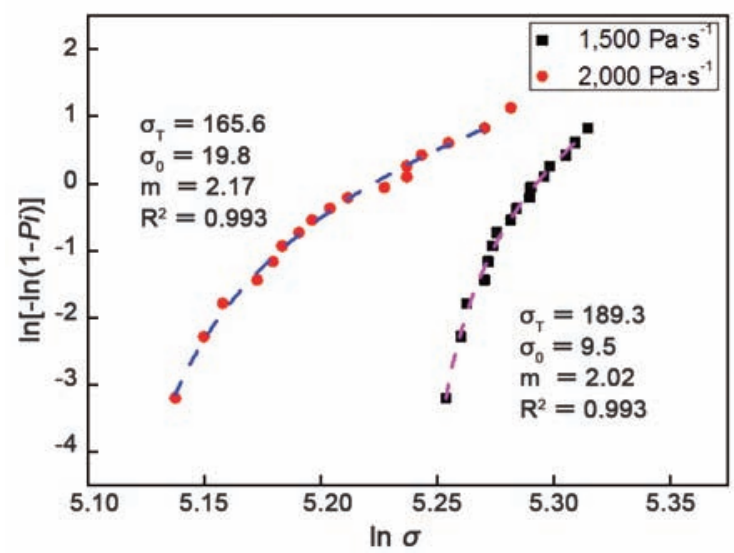

Fig. 10: Weibull distributions of ultimate tensile strengths
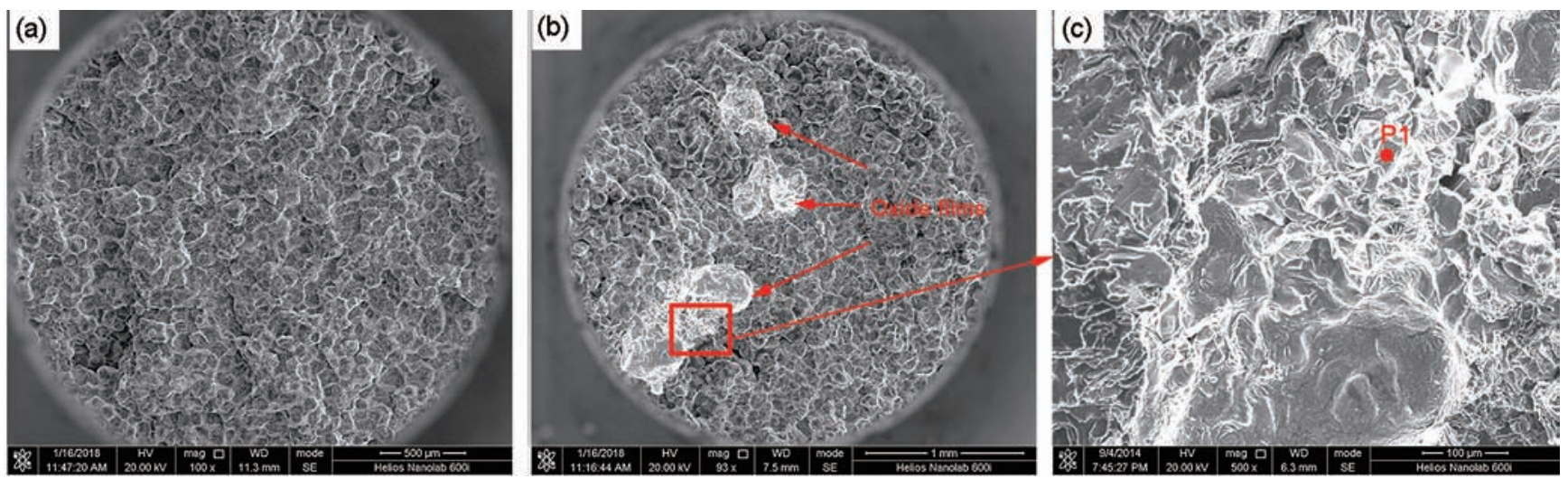

Fig. 11: Fracture morphologys with different pressuring speeds: (a)1,500 $\mathrm{Pa}^{-1} \mathrm{~s}^{-1}$, (b) 2,000 $\mathrm{Pa} \cdot \mathrm{s}^{-1}$, (c) magnification of red box in (b)

observed in the both specimens with pressurizing speeds of 1,500 $\mathrm{Pa} \cdot \mathrm{s}^{-1}$ and 2,000 $\mathrm{Pa} \cdot \mathrm{s}^{-1}$, respectively. For ZL205A alloy, the crystallization range is about $90 \mathrm{~K}^{[14]}$ and the fluidity is poorer than Al-Si system alloys. Thus, the shrinkage cavity in the castings of ZL205A alloy is almost difficult to avoid. Oxide film defects were found on the fracture surfaces of the specimens with pressurizing speeds of 2,000 $\mathrm{Pa} \cdot \mathrm{s}^{-1}$. The EDX analysis result shows that the oxide film contains oxygen element with the content of $14.5 \%$, as shown in Table 3 .

A double oxide film defect can acts as a crack, causing a major reduction in strength ${ }^{[2]}$. The effects of these defects on mechanical properties are difficult to measure qualitatively. Weibull distribution can be used to characterize the distribution

Table 3: EDX spectra obtained from point P1 given in Fig. 11 (c)

\begin{tabular}{ccc} 
Element & Wt.\% & At.\% \\
\hline $\mathrm{O}$ & 14.57 & 19.47 \\
$\mathrm{Cu}$ & 5.33 & 17.34 \\
$\mathrm{Al}$ & 78.82 & 62.53 \\
$\mathrm{Fe}$ & 1.28 & 0.64
\end{tabular}


of the fracture strength of castings ${ }^{[15,16]}$. The scatter in tensile strength was controlled by the level of the defects contained, which appear to directly decrease the threshold value of the castings.

Obviously, oxide film entrainment occurred when the pressurizing speed was $2,000 \mathrm{~Pa} \cdot \mathrm{s}^{-1}$ and caused a reduction in the threshold value of tensile properties. While the pressurizing speed was $1,500 \mathrm{~Pa} \cdot \mathrm{s}^{-1}$, the filling process was steady and smooth in this work. The experiment results indicate that the critical state calculated from Eq. (6) is accurate.

\section{Conclusions}

(1) The filling process in a gradual expansion structure during LPC can be divided into spreading stage and filling stage. The gate velocity continues to increase in the whole mold filling process. The main reason is that the increasing cross-section area of cavity reduces the raising of liquid level under a constant pressurizing speed.

(2) The gate velocity increases with the expansion angle and pressurizing speed during the filling process of a gradual expansion structure in LPC.

(3) Differenting from the filling process of a sudden expansion structure, oxide film entrainment does not necessarily occur when the gate velocity is greater than critical velocity in the gradual expansion structure filling process, due to the velocity of the fluid decreasing in the jet zone from ingate to free surface.

(4) To avoid the entrainment of oxide filling, a mathematical model between the pressurizing speed, expansion angle and the height of the gradual expansion structure was established. According to which, the reasonable pressurizing speed can be determined.

\section{References}

[1] Cox M, Harding R A, Green N R, et al. Influence of vacuum counter-gravity filling on reliability of 2L99 aluminium investment castings. Mater. Sci. Technol., 2007, 23(9): 1075-
1084

[2] Campbell J. Castings. Oxford, UK: Butterworth-Heinemann, 2003: 17.

[3] Fox S, Campbell J. Visualisation of oxide film defects during solidification of aluminium alloys. Scripta Mater., 2000, 43(10): 881-886.

[4] Mi J, Harding R A, Wickins M, Campbell J. Entrained oxide films in TiAl castings. Intermetallics, 2003, 11: 377-385.

[5] Afsharpour M, Homayun B, Boutorabi S M A. Water modelling of effects of pouring basin and sprue geometry on entrance of air bubbles into mould. Mater. Sci. Eng., A, 2014, 30(2): 152159.

[6] Runyoro J, Boutorabi S M A, Campbell J. Critical gate velocities for film-forming casting alloys: A basis for process specification. Trans. Am. Foundry Soc., 1992, 37: 225-234

[7] Bahreian F, Boutorabi S M A, Campbell J. Critical gate velocity for magnesium casting alloy (ZK51A). Int. J. Cast met. Res. 2006, 19(1): 45-51.

[8] Campbell J. Castings. Oxford, UK: Butterworth-Heinemann, 1991: 66-68.

[9] Liu Shanguang, Cao Fuyang, Zhao Xinyi, et al. Characteristics of mold filling and entrainment of oxide film in low pressure casting of A356 alloy. Mater. Sci. Eng., A 2015;626: 159-164.

[10] Liu Shanguang, Cao Fuyang, Ying Tao, et al. Formation Mechanism of Surface Crack in Low Pressure Casting of A360 Alloy. Metall. Mater. Trans. B, 2017, 48(6): 2826-2835.

[11] Kawaguchi Y, Segawa T, Feng Z, et al. Experimental study on drag-reducing channel flow with surfactant additives spatial structure of turbulence investigated by PIV system. Int. J. Heat Fluid, 2002, 23: 700-709.

[12] Tiryakioglu M, Campbell J. Weibull Analysis of Mechanical Data for Castings: A Guide to the Interpretation of Probability Plots. Metall. Mater. Trans. A, 2010, A41: 3121-3129

[13] Liu Shanguang, Cao Fuyang, Yi Junying, et al. Effect of depressurizing speed on mold filling behavior and entrainment of oxide film in vacuum suction casting of A 357 alloy. Trans. Nonferrous Met. Soc. China, 2016: 3292-3298.

[14] A. Zhang, S. Liang, Z. Guo, S. Xiong, Determination of the interfacial heat transfer coefficient at the metal-sand mold interface in low pressure sand casting, Exp Therm Fluid Sci 88 (2017) 472-482.

[15] Green N R, Campbell J. Influence of Oxide film Filling Defects of Al-7Si-Mg alloy Castings. AFS Trans, 1994, 102:341-347.

[16] Mi J, Harding R A, Campbell J. Effect of the Entrained Surface film on the Reliability of Castings. Metall. Mater. Trans. A, 2004, 35A: 2893-2902. 